\title{
Association of Internet gaming disorder symptoms with anxiety and depressive symptoms and substance use: an international cross-sectional study
}

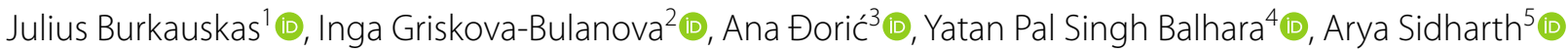 \\ , Ramdas Ransing ${ }^{6} \mathbb{B}$, Tuong -. Vi Vu Thi ${ }^{7}$, Truong Ngoc Huong ${ }^{8}$, Helin Yilmaz Kafali ${ }^{9}$, Gamze Erzin ${ }^{10}{ }^{\infty}$ \\ , Zahir Vally ${ }^{11}$, Mita Rani Roy Chowdhury ${ }^{12}$, Pawan Sharma ${ }^{13}$, Rabi Shakya ${ }^{13}$, Paulo Moreira ${ }^{14}$ (D), \\ Sara Faria ${ }^{14}$, Isa Multazam Noor ${ }^{15}$, Luís Antônio Monteiro Campos ${ }^{16}$ (D) Anna Rebeka Szczegielniak ${ }^{17}$ (1) and \\ Dejan Stevanovic ${ }^{18^{*}}$ (D)
}

\begin{abstract}
Background: Problematic Internet gaming is an increasingly recognized global mental health problem. This multicultural cross-sectional study examined the association between Internet gaming disorder (IGD) symptoms and anxiety and depressive symptoms and substance use within a sample of young Internet users. In total, 3529 college/ university students (1260 (35.7\%) males; mean age $21 \pm 3$ years) were surveyed online. We assessed online gaming patterns using the Internet Gaming Disorder Self-report for College/University Students (ICMH-IGD), symptoms of depression using the Patient Health Questionnaire-9, and symptoms of anxiety using the Generalized Anxiety Disorder scale-7.

Results: IGD symptoms were associated with symptoms of depression, anxiety, and substance use, independent of time spent online, psychiatric diagnosis, culture, or sociodemographic characteristics. For males, more significant IGD symptoms were associated with more extended Internet browsing per day time and higher levels of anxiety and depressive symptoms, while for females, with more extended Internet browsing per day time, marihuana use, and higher levels of depressive symptoms.
\end{abstract}

Conclusions: Our study found that more overt symptoms of IGD were associated with higher levels of anxiety and depressive symptoms and substance use. Still, these associations differed among males and females, suggesting that gender differences should be considered when planning specific treatments.

Keywords: Problematic gaming, Gaming addiction, Gaming disorder, Psychiatric symptoms, Substance abuse, Anxiety, Depression

\footnotetext{
*Correspondence: stevanovic.dejan79@gmail.com

${ }^{18}$ Clinic for Neurology and Psychiatry for Children and Youth, Dr. Subotic

$6 a$, Belgrade 11000, Serbia

Full list of author information is available at the end of the article
}

\begin{abstract}
Background
Problematic Internet gaming is an increasingly recognized global mental health problem [1-3]. In 2013, Internet gaming disorder (IGD) was included in the Diagnostic and Statistical Manual (DSM-5) as a candidate disorder requiring future research [4], while the International Classification of Diseases (ICD) 11th
\end{abstract}


edition recognizes gaming disorder as a new category which includes online gaming as well as offline gaming [5]. IGD is mainly defined as a condition characterized by an increased priority given to gaming, which takes precedence over other life interests and daily activities, and results in impaired control and continuation of/ or increase in gaming despite its negative consequences [6]. These core features of IGD resemble those observed in other behavioral addictions, such as substance-related disorders $[7,8]$. Although there is an increased interest in IGD research and even treatment approaches are suggested [9-11], large-scale cross-national studies evaluating possible contributors to IGD symptoms are scarce, and many of them focus on adolescents [12] or children [13]. However, it has been shown that young adults are especially vulnerable to IGD $[14,15]$. Therefore, studies aiming at the identification of factors affecting the problematic online gaming in young adults are needed to promote better recognition of individuals at risk for IGD [12, 16 , 17] and, as a consequence, faster interventions [18] and better management of symptoms related to IGD, especially facing possible mental health disturbances due to COVID-19 pandemic [19].

Depressive and anxiety symptoms have been linked to poor outcomes across different individuals engaged in problematic Internet use [20]. Recent studies have also suggested that IGD may be closely associated with depression, based on the overlapping neural mechanisms in depressed individuals and those with IGD [21-23]. For example, a community health survey of 190,066 participants conducted in Korea in 2017 found that IGD is associated with depression, impairments of daily activities, reduced quality of life, and suicidal ideation and suicide attempts [24]. Similarly, another Korean study comprising 7200 survey participants found that depression was a common comorbid condition among individuals with IGD and was associated with severe clinical phenomenology, including worsened symptoms of alcohol and nicotine addiction and a generalized anxiety disorder [25]. Indeed, other psychological conditions such as generalized anxiety disorder have also been a common comorbid disorder in IGD [26]. Another study comprising a representative sample of 1531 teenagers and young adults found associations between IGD and higher depressive and anxiety symptoms [27]. On a different note, IGD is often associated with various substance use disorders. For example, a recent review suggested that adults with disordered gaming frequently show problematic or disordered substance use [28]. In addition, several studies analyzing neuroanatomical changes in the brain, including reward and executive functioning mechanisms, have reported similar changes in both IGD and substance use [24, 29-32].
Finally, a notable aspect of IGD studies is gender differences. A recent meta-analysis on gaming patterns in 82,440 participants across 21 countries has confirmed that higher levels of IGD exist in males than females [33]. This might result from several reasons, including differences in neural mechanisms related to craving [34], temperamental [35], or socio-cultural factors [36], and coping strategies [37]. While many studies report gender differences in IGD symptomatology, their relation to possible comorbidities is still unknown and requires further investigation. For example, a survey of 429 French online gamers (mean age 21 years) found that in male subjects, high anxiety and depression scores were associated with IGD, while for females, only depression scores were associated with IGD [38].

Further studies are needed to understand better the interplay between symptoms of anxiety, depression, and substance use in IGD, taking into account gender differences. Thus, this study aimed to investigate the relationship between depression, anxiety, and substance use with IGD symptoms, using an international, multicultural sample of Internet users who completed an online survey. Considering previous studies, we hypothesized to find gender differences in IGD symptoms. In addition, it was expected that depression and anxiety symptom severity would be associated with more significant IGD symptoms. Finally, we had an exploratory aim to investigate the association of substance use with IGD symptom severity.

\section{Methods}

\section{Participants}

We used the data for the present study from an already available database $[39,40]$. The data were collected from students pursuing various graduation courses in colleges and universities across 15 countries, throughout the International Child Mental Health Study Group (ICMHSG; http://www.icmhsg.org), including Bangladesh, Brazil, Croatia, India, Indonesia, Italy, Poland, Portugal, Nepal, Nigeria, Serbia, Sweden, Turkey, Vietnam, and the United Arab Emirates (UAE). The lead authors from different countries were responsible for advertising the study in the respective countries, soliciting the students directly or via students' organizations, and sending a link to the survey. It was considered a mode of convenience sampling and the engagement rate was not monitored. Before completing the survey, each participant was formally asked to provide online consent. No incentives were given upon completion.

An online survey using a cross-sectional design was considered for the data collection due to its benefits of accessing larger sample pools and reaching heterogeneous groups, its cost-efficiency, and its practical 
advantages for researching behavioral addictions [39-41]. The online survey with information about the study (i.e., objectives and assurances of anonymity and confidentiality) and the instruments (see below) was made available from February 2018 until June 2019 using Google Forms. The survey questionnaire was prepared and made available in additional languages apart from English for the data collection in the countries (i.e., Croatian, Indonesian, Polish, Portuguese, Serbian, Turkish, and Vietnamese, respectively) for students unfamiliar with the English language. For additional details on the sampling and procedures, the reader is referred to $[39,40]$.

The present study received approvals from the ethics committees of all relevant countries and conformed to the principles outlined in the Declaration of Helsinki.

\section{Instruments}

The online survey included the following instruments. Sociodemographic information, as well as information on substance use and previous psychiatric diagnoses, was collected first. Next, substance use was assessed with a single question on particular substance use (e.g., alcohol, marihuana, and others) over the past month on a 5-point Likert scale from 0 "no usage" to 5 "using substance almost every day."

\section{The International Child Mental Health Study Group Internet Gaming Disorder Self-report for College/University Students (ICMH-IGD)}

The ICMH-IGD is a self-report instrument developed and validated by the International Child Mental Health Study Group to assess problematic online gaming activity [40]. The instrument comprises 11 items rated on a five-point Likert scale (i.e., one "never" to five "always"), where a higher score indicates a greater difficulty. The scale development adheres to the main requirements for measuring IGD [42]. For example, the scale covers all DSM-5 IGD criteria and has good validation sample quality, indicated by psychometric properties evaluated in nationally representative samples. The sum score of the instrument ranges from nine to 45 , with higher scores indicating a greater intensity of IGD symptoms present. The instrument was previously validated for the English, Croatian, Polish, Portuguese, Serbian, Turkish, and Vietnamese languages, with good psychometric characteristics demonstrated [40]. The Indonesian version was also psychometrically evaluated and its Cronbach's $\alpha$ was 0.88 . The Cronbach's $\alpha$ of the instrument in the current sample was 0.92 .

\section{Generalized Anxiety Disorder scale-7 (GAD-7)}

The GAD-7 a self-report instrument, comprises seven items scored from zero to three, with total scores ranging from zero to 21 with a higher score indicating greater anxiety symptoms severity [43]. In addition, the GAD-7 instrument has solid internal and test-retest reliability and construct validity [43]. The Cronbach's $\alpha$ of the GAD-7 in the current sample was 0.90 .

\section{Patient Health Questionnaire-9 (PHQ-9)}

The PHQ-9 is a self-report instrument developed for fast and efficient psychiatric screening for depression symptoms [44]. The instrument includes nine items on depression severity scored from zero to three, with total scores varying from zero to 27, where higher scores indicate more significant depressive symptomatology. The Cronbach's $\alpha$ of the PHQ-9 in the current sample was 0.87 .

\section{Statistical analysis}

We expressed qualitative data as percentages and quantitative data as means \pm standard deviation. The following substance use groups were considered: depressant drugs (i.e., sedatives or tranquilizers, heroin, painkillers, marijuana, and inhalants or solvents), stimulant drugs (i.e., cocaine, methamphetamines, and other stimulants), hallucinogen drugs (i.e., hallucinogen and club drugs), and alcohol. For all variables, we performed normality tests, including skewness, kurtosis, and one-sample Kolmogorov-Smirnov tests, and found no violations of the normal distribution. Differences in sociodemographic, depression, anxiety, substance use and gaming characteristics of participants, stratified by gender, were tested using independent sample $t$-tests, the Pearson chi-square test, and in cases where the $p$-value was lower than .05 difference the between proportions test. Next, we performed multiple linear regression analyses to determine whether depression, anxiety symptom severity, and substance use were independently associated with IGD severity after adjustment for sociodemographic factors, previous psychiatric diagnosis, culture (countries were coded as dummy variables), and time spent online (i.e., number of hours per day). Thus, we designed five separate multiple regression models, including symptoms of depression, anxiety, and substance use variables (i.e., the PHQ-9, GAD-7, type of substance) as predictors of IGD symptoms severity. We examined scatterplots of residuals to check the assumptions of the regression analysis: normality, linearity, and homoscedasticity. The variance inflation factor and tolerance statistic indicated no problem with multicollinearity; all variance inflation factor values were $<2.4$. We performed Bonferroni corrections for multiple regression analyses to reduce the likelihood of type I error. We pre-assigned a $p$-value as the threshold for significance by adjusting the $p$-value for the number of statistical comparisons: 
$p$ - value of $0.05 \div$ number of multiple regression models

Hence, the corrected $p$-value was $0.05 \div 5=0.01$

We required $p<0.01$ for a predictor to be considered as significant.

Structural equation modeling (SEM) examined the relationships between alcohol abuse, hallucinogenic drugs, stimulant drugs, depressant drugs, depressive, anxiety, and IGD symptoms. Three models were performed to evaluate those relationships in all the collected and male and female samples. We used the SPSS 17.0 for Windows statistical package (SPSS Inc., Chicago, Illinois) for all analyses and software AMOS.

\section{Results}

Data from 3529 subjects (1260 (35.7\%) males; mean age $21 \pm 3$ years) were available. As demonstrated in Table 1 , significant differences were found between males and females in the respective instruments for IGD, anxiety, depression, and the frequency of marihuana and other reported substance use. In total, 79 (2.3\%) individuals reported using stimulants, 202 (5.8\%) sedatives or tranquilizers, 977 (27.8\%) alcohol, 195 (5.5\%) marijuana, 67 (1.9\%) cocaine/crack, 63 (1.8\%) club drugs, 59 (1.7\%) hallucinogens, 55 (1.6\%) heroin, 66 (1.8\%) inhalants or solvents, 64 (1.8\%) methamphetamines, and 595 (16.8\%) painkillers (details available in a supplementary file).

Table 2 shows the multiple linear regression analyses for males, which revealed that the total number of hours of Internet browsing per day $(\beta=0.14)$, anxiety symptoms $(\beta=0.09)$, and depression symptoms $(\beta=0.17)$ were significantly associated with more significant IGD symptoms, predicting $16.1 \%$ of the variance, when adjusted for culture and previous psychiatric diagnosis.

Table 3 shows the multiple linear regression analyses for females, which revealed that the total number of

Table 1 Characteristics of all participants

\begin{tabular}{|c|c|c|c|c|c|}
\hline \multirow[t]{2}{*}{ Characteristics } & \multicolumn{3}{|l|}{ Participants } & \multirow[t]{2}{*}{$P$} & \multirow{2}{*}{$\begin{array}{l}\text { Difference } \\
\text { between } \\
\text { proportions, \% }\end{array}$} \\
\hline & All $(N=3529)$ & Males $(n=1260)$ & Females $(n=2269)$ & & \\
\hline Age & $21.30 \pm 2.74$ & $21.54 \pm 2.89$ & $21.17 \pm 2.64$ & $<0.001$ & \\
\hline Previous psychiatric diagnosis & $219(6.2 \%)$ & $75(6.0 \%)$ & $144(6.3 \%)$ & 0.642 & $0.3 \%$ \\
\hline Hours of Internet browsing per day & $5.71 \pm 4.47$ & $5.57 \pm 4.28$ & $5.79 \pm 4.58$ & 0.153 & \\
\hline Alcohol use & & & & 0.125 & \\
\hline Not at all & $2552(72.3 \%)$ & $878(69.7 \%)$ & $1674(73.8 \%)$ & & $4.1 \%$ \\
\hline One or 2 days & $737(20.9 \%)$ & $284(22.5 \%)$ & $453(20.0 \%)$ & & $2.5 \%$ \\
\hline Several days & $186(5.3 \%)$ & $76(6.0 \%)$ & $110(4.8 \%)$ & & $1.2 \%$ \\
\hline More than half the days & $34(1.0 \%)$ & $14(1.1 \%)$ & $20(0.9 \%)$ & & $0.2 \%$ \\
\hline Nearly every day & $20(0.6 \%)$ & $8(0.6 \%)$ & $12(0.5 \%)$ & & $0.1 \%$ \\
\hline Marihuana use & & & & 0.006 & \\
\hline Not at all & 3334 (94.5\%) & $1166(92.5 \%)$ & $2168(95.5 \%)$ & & $3.0 \%$ \\
\hline One or 2 days & $117(3.3 \%)$ & $55(4.4 \%)$ & $62(2.7 \%)$ & & $1.7 \%$ \\
\hline Several days & $45(1.3 \%)$ & $22(1.7 \%)$ & $23(1.0 \%)$ & & $0.7 \%$ \\
\hline More than half the days & $11(0.3 \%)$ & $6(0.5 \%)$ & $5(0.2 \%)$ & & $0.3 \%$ \\
\hline Nearly every day & $22(0.6 \%)$ & $11(0.9 \%)$ & $11(0.5 \%)$ & & $0.4 \%$ \\
\hline Other substances use & $728(20.6 \%)$ & $222(17.6 \%)$ & $506(22.3 \%)$ & 0.001 & $4.7 \%$ \\
\hline GAD-7 score & $6.17 \pm 4.96$ & $5.78 \pm 4.93$ & $6.39 \pm 4.97$ & $<0.001$ & \\
\hline PHQ-9 score & $7.98 \pm 5.63$ & $7.62 \pm 5.58$ & $8.19 \pm 5.65$ & 0.004 & \\
\hline ICMH-IGD total sample score & $13.30 \pm 6.03$ & $15.45 \pm 6.81$ & $12.11 \pm 5.19$ & $<0.001$ & \\
\hline Croatian sample score & $437(12.4 \%)$ & $104(8.3 \%)$ & $333(14.7 \%)$ & $<0.001$ & $6.4 \%$ \\
\hline Serbian sample score & $316(9.0 \%)$ & $91(7.2 \%)$ & $225(9.9 \%)$ & 0.007 & $2.7 \%$ \\
\hline Portuguese sample score & $126(3.6 \%)$ & $31(2.5 \%)$ & $95(4.2 \%)$ & 0.008 & $1.7 \%$ \\
\hline Turkish sample score & $244(6.9 \%)$ & $94(7.5 \%)$ & $150(6.6 \%)$ & 0.341 & $0.9 \%$ \\
\hline Vietnamese sample score & $426(12.1 \%)$ & $141(11.2 \%)$ & $285(12.6 \%)$ & 0.231 & $1.4 \%$ \\
\hline Polish sample score & $156(4.4 \%)$ & $43(3.4 \%)$ & $113(5.0 \%)$ & 0.030 & $1.6 \%$ \\
\hline Indonesian sample score & $673(19.1 \%)$ & $188(14.9 \%)$ & $485(21.4 \%)$ & $<0.001$ & $6.5 \%$ \\
\hline English sample score & $1151(32.6 \%)$ & $568(45.1 \%)$ & $583(25.7 \%)$ & $<0.001$ & $19.4 \%$ \\
\hline
\end{tabular}

$p$-value calculated Student's $t$-test for continuous variables and the $\chi^{2}$ test or Fisher's exact test for categorical variables 
Table 2 Significant determinants of Internet gaming disorder self-report for college/university students by the International Child Mental Health Study Group, ICMH-IGD Scales, in 1260 male students

\begin{tabular}{|c|c|c|c|c|c|c|c|c|c|c|c|}
\hline & & ICMH-IGI & score & PHQ-9 sc & ore & GAD-7 s & ore & Alcohol & use & Marihu & ana use \\
\hline & & $\beta$ & & $\beta$ & & $\beta$ & & $\beta$ & & $\beta$ & \\
\hline Model 1 & $R^{2}$ & & $0.007^{* *}$ & & $0.008^{* *}$ & & $0.005^{*}$ & & $0.034^{* * *}$ & & 0.000 \\
\hline & $\Delta R^{2}$ & & $0.007^{* *}$ & & $0.008^{* *}$ & & $0.005^{*}$ & & $0.034^{* * *}$ & & 0.000 \\
\hline Age & & $-0.08^{* *}$ & & $-0.09^{* *}$ & & $-0.07^{*}$ & & $0.19^{* * *}$ & & 0.02 & \\
\hline Model 2 & $R^{2}$ & & $0.096 * * *$ & & $0.094^{* * *}$ & & $0.092^{* * *}$ & & $0.161^{* * *}$ & & $0.039^{* * *}$ \\
\hline & $\Delta R^{2}$ & & $0.089^{* * *}$ & & $0.086^{* * *}$ & & $0.088^{* * *}$ & & $0.127^{* * *}$ & & $0.039^{* * *}$ \\
\hline Age & & -0.04 & & $-0.08^{* *}$ & & $-0.08^{* *}$ & & $0.13^{* * *}$ & & -0.01 & \\
\hline Previous psychiatric diagnosis & & 0.04 & & $0.18^{* * *}$ & & $0.22^{* * *}$ & & $0.09^{* * *}$ & & $0.06^{*}$ & \\
\hline Hours of Internet browsing per day & & $0.19^{* * *}$ & & $0.16^{* * *}$ & & $0.17^{* * *}$ & & $0.11^{* * *}$ & & 0.04 & \\
\hline Model 3 & $R^{2}$ & & $0.161^{* * *}$ & & $0.546^{* * *}$ & & $0.544^{* * *}$ & & $0.255^{* * *}$ & & $0.153^{* * *}$ \\
\hline & $\Delta R^{2}$ & & $0.065^{* * *}$ & & $0.452^{* * *}$ & & $0.452^{* * *}$ & & $0.094^{* * *}$ & & $0.114^{* * *}$ \\
\hline Age & & -0.02 & & -0.02 & & -0.03 & & $0.13^{* * *}$ & & -0.03 & \\
\hline Previous diagnosis & & -0.02 & & 0.02 & & $0.09^{* * *}$ & & $0.07^{*}$ & & 0.01 & \\
\hline Hours of Internet browsing per day & & $0.14^{* * *}$ & & 0.04 & & $0.05^{*}$ & & $0.09^{* *}$ & & 0.02 & \\
\hline Alcohol use & & 0.04 & & -0.01 & & 0.01 & & & & $0.30^{* * *}$ & \\
\hline Marihuana use & & 0.05 & & 0.01 & & 0.03 & & $0.26^{* * *}$ & & & \\
\hline Other substances & & 0.05 & & $0.06^{* *}$ & & 0.04 & & $0.13^{* * *}$ & & $0.13^{* * *}$ & \\
\hline GAD-7 score & & $0.09^{* *}$ & & $0.69^{* * *}$ & & & & 0.01 & & 0.05 & \\
\hline PHQ-9 score & & $0.17^{* * *}$ & & & & $0.70^{* * *}$ & & 0.02 & & 0.03 & \\
\hline
\end{tabular}

GAD-7 Generalized Anxiety Disorder scale-7, PHQ-9 Patient Health Questionnaire-9, ICMH-IGD Internet Gaming Disorder Self-report for College/University Students by the International Child Mental Health Study Group

${ }^{*} p$ value $<.05 ;{ }^{* *} p$ value $<.01 ;{ }^{* * *} p$ value $<.001$ adjusted for culture

hours of Internet browsing per day $(\beta=0.13)$, marihuana use $(\beta=0.10)$, and depression symptoms $(\beta=0.21)$ were significantly associated with more significant IGD symptoms, predicting $17.6 \%$ of the variance, when adjusted for culture and previous psychiatric diagnosis.

\section{Structural equation models}

The model shown in Fig. 1 examined the relationships between alcohol abuse, stimulant drugs, depressant drugs, hallucinogens drugs, depressive, anxiety, and IGD symptoms in the whole sample. Alcohol abuse was positively associated depressive $(\beta=0.24, \mathrm{CI} 95 \%[0.21 ; 0.27]$, $p<0.001)$ and anxiety symptoms $(\beta=0.34$, CI $[0.31 ; 0.37]$, $p<0.001)$. Depressant drugs were positively associated with depressive $(\beta=0.13$, CI95\% $[0.8 ; 0.19], p<0.001)$ and anxiety symptoms $(\beta=0.08$, CI95\% [0.03; 0.13$]$, $p=0.004)$. Stimulant drugs were negatively associated with anxiety symptoms $(\beta=-0.08$, CI95\% $[-0.13 ;-0.02]$, $p=0.002$ ), hallucinogen drugs with depressive symptoms $(\beta=-0.09$, CI95\% $[-0.14 ;-0.03], p=0.002)$, and with IGD symptoms $(\beta=0.17$, CI95\% [0.14; 0.21$], p<0.001)$. Depressive symptoms were positively associated with IGD symptoms ( $\beta=0.24$, CI95\% [0.20; 0.27], $p<0.001)$.

The model shown in Fig. 2 examined the relationships between alcohol abuse, stimulant drugs, depressant drugs, hallucinogens drugs, depressive, anxiety, and IGD symptoms in males. Alcohol abuse was positively associated depressive $(\beta=0.25$, CI95\% [0.19;0.30], $p<0.001)$ and anxiety symptoms $(\beta=0.38$, CI95\% [0.34; 0.43$]$, $p<0.001)$. Depressant drugs were positively associated with depressive symptoms $(\beta=0.15$, CI95\% [0.06; $0.25], p<0.001)$, and IGD $(\beta=0.15$, CI95\% [0.05; 0.26], $p=0.004)$. Stimulant drugs were negatively associated with IGD symptoms $(\beta=-0.20$, CI95\% [ $-0.35 ;-0.05]$, $p=0.002$ ), hallucinogen drugs with depressive symptoms $(\beta=-0.12$, CI95\% $[-0.21 ;-0.03], p=0.012)$, but positively associated with IGD $(\beta=0.17$, CI95\% [0.02; 0.32], $p=0.022)$. Depressive symptoms were positively associated with IGD symptoms $(\beta=0.21$, CI95\% [0.13; 0.28$]$, $p<0.001)$ as well as anxiety symptoms $(\beta=0.09$, CI95\% [0.02; 0.17], $p=0.01$ ).

The model shown in Fig. 3 examined the relationships between alcohol abuse, stimulant drugs, depressant drugs, hallucinogens drugs, depressive, anxiety, and IGD symptoms in females. Alcohol abuse was positively associated depressive $(\beta=0.24, \quad$ C195\% $[0.19 ; 0.28], p<0.001)$ and anxiety symptoms $(\beta=0.31$, CI95\% [0.27; 0.35], $p<0.001)$. Depressant drugs were positively associated with depressive $(\beta=0.07$, CI95\% $[0.03 ; .11], p<0.001)$ and anxiety symptoms $(\beta=0.11$, 
Table 3 Significant determinants of Internet gaming disorder self-report for college/university students by the International Child Mental Health Study Group, ICMH-IGD Scales, in 2269 female students

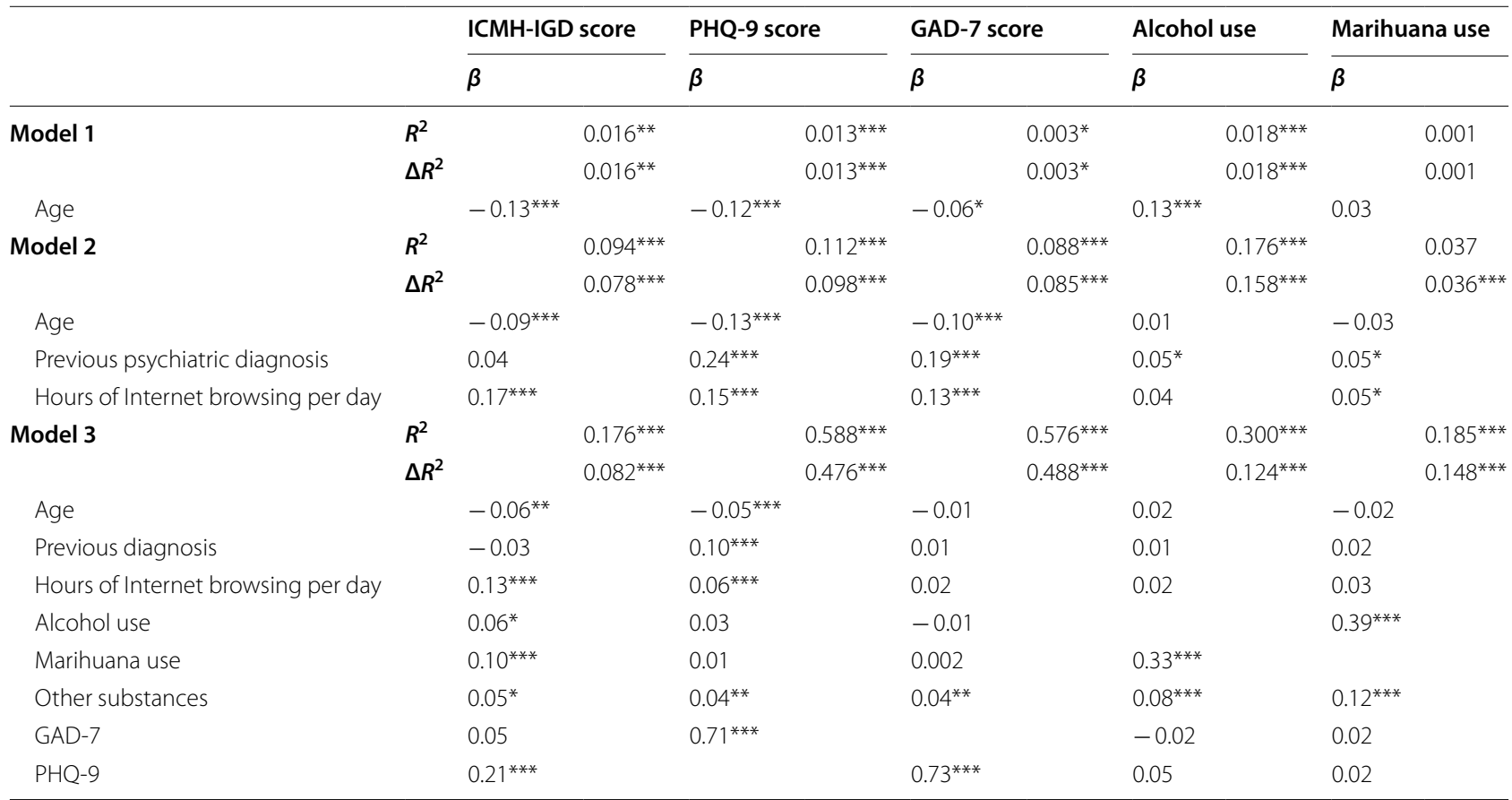

GAD-7 Generalized Anxiety Disorder scale-7, PHQ-9 Patient Health Questionnaire-9, ICMH-IGD Internet Gaming Disorder Self-report for College/University Students by the International Child Mental Health Study Group

${ }^{*} p$ value $<.05 ;{ }^{* *} p$ value $<.01 ;{ }^{* * *} p$ value $<.001$ adjusted for culture

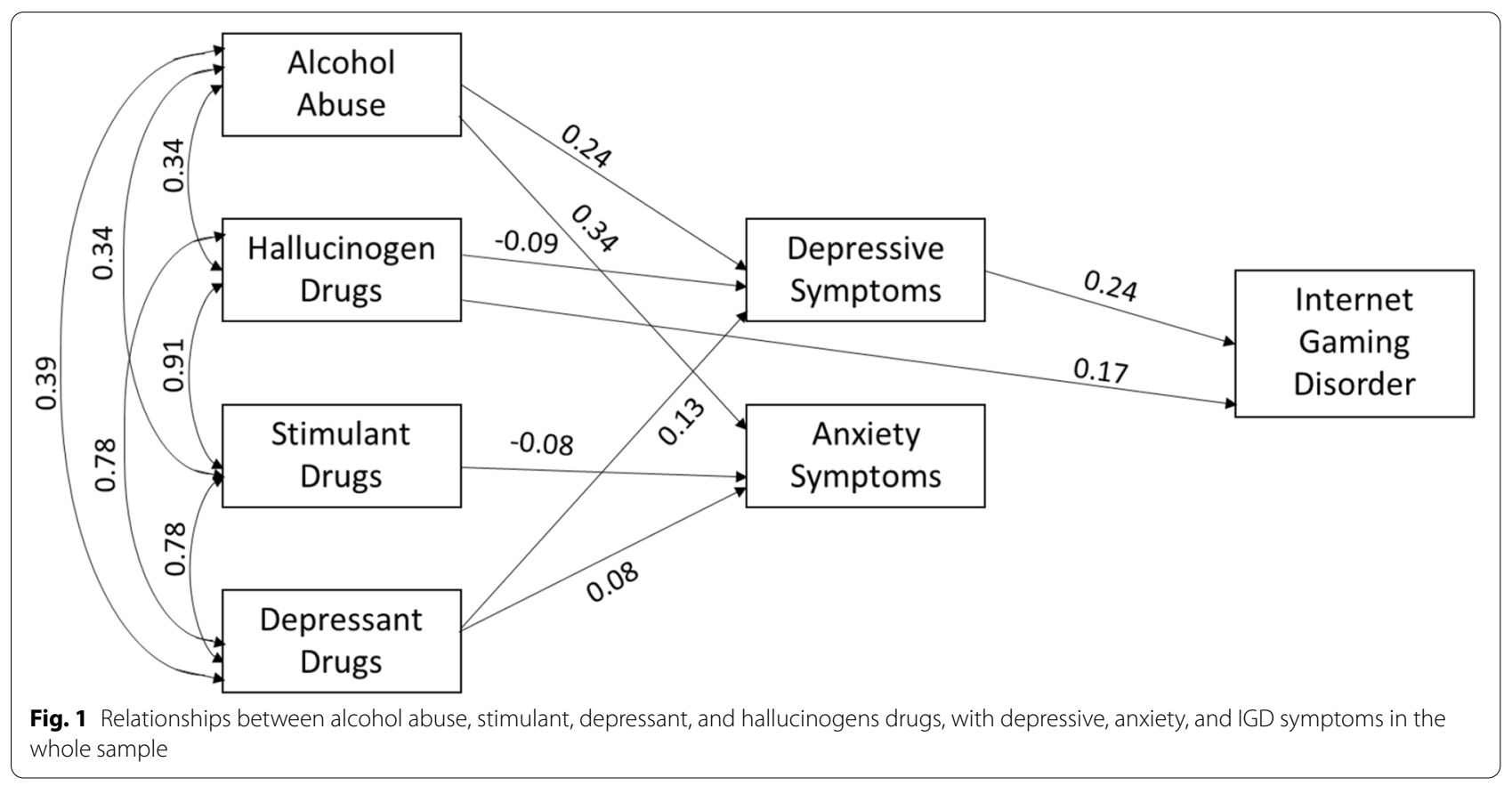




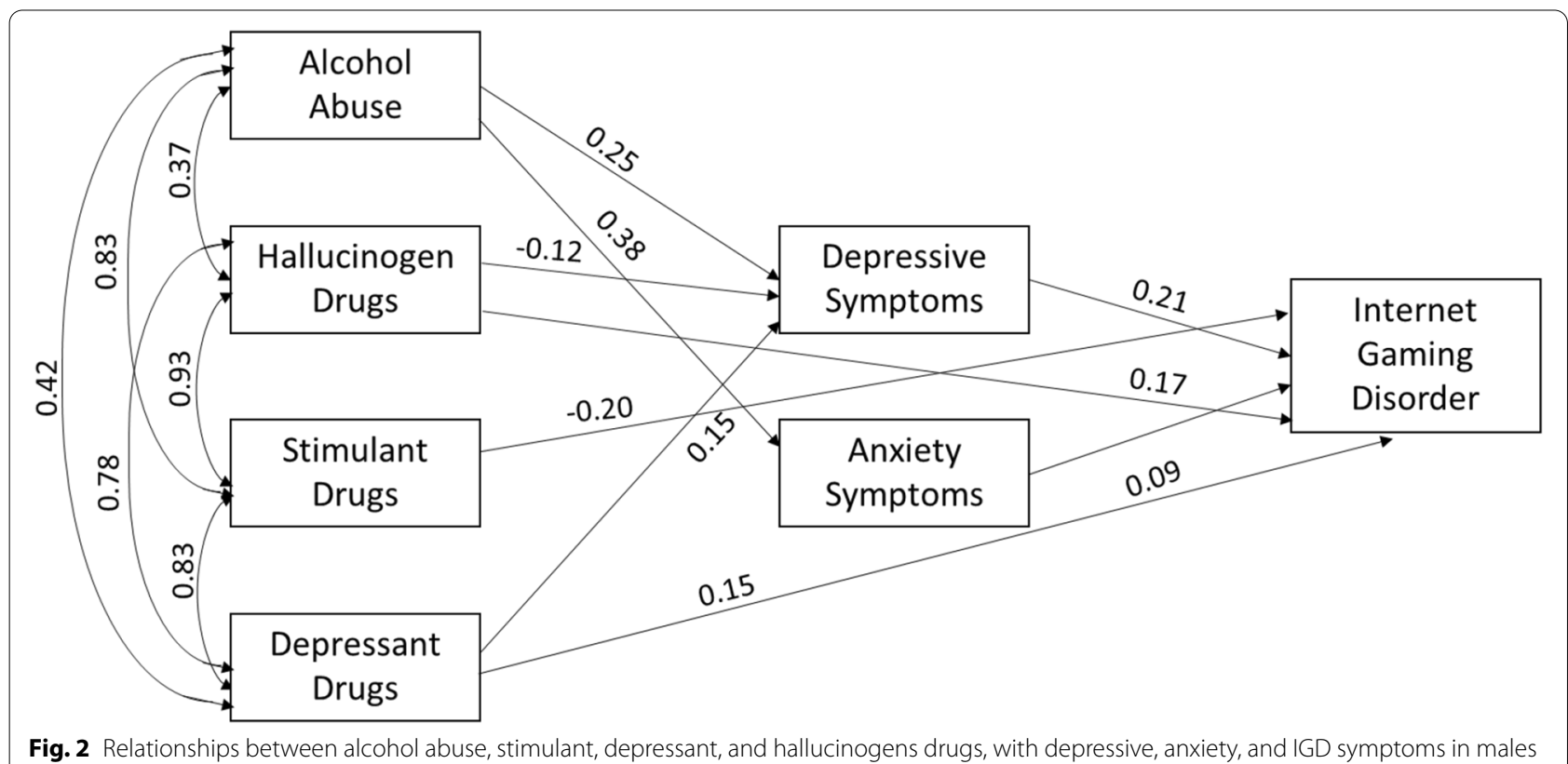

Fig. 2 Relationships between alcohol abuse, stimulant, depressant, and hallucinogens drugs, with depressive, anxiety, and IGD symptoms in males

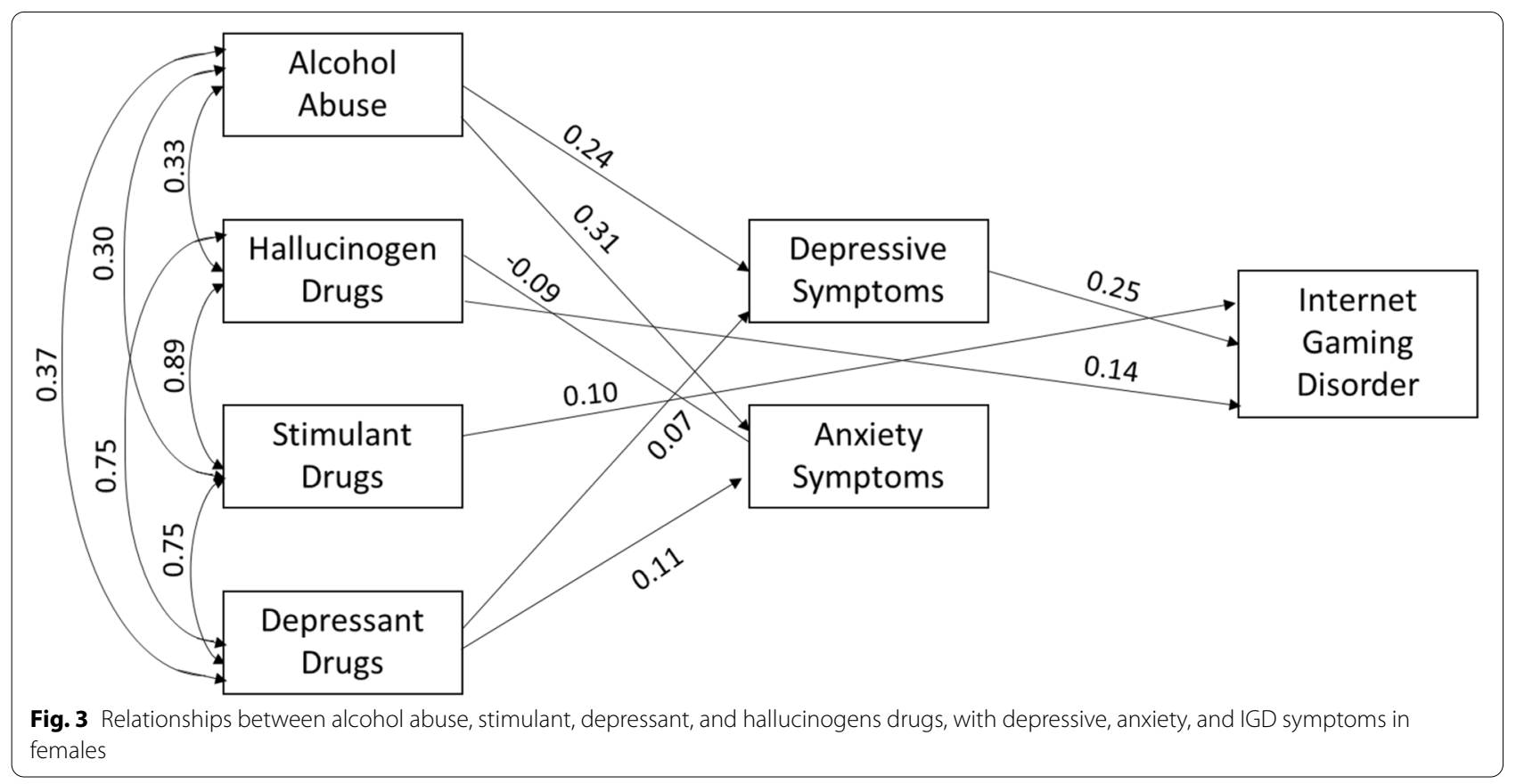

CI95\% [0.05; 0.17], $p<0.001)$. Stimulant drugs were positively associated with IGD symptoms $(\beta=-0.10$, CI95\% $[-0.35 ;-0.05], p=0.002)$. Hallucinogen drugs were negatively associated with anxiety $(\beta=-0.08$, CI95\% $[-0.15 ;-0.03], p=0.004)$, but positively with IGD symptoms $(\beta=0.14$, CI95\% [0.06; 0.23], $p<0.001)$. Depressive symptoms were positively associated with IGD symptoms ( $\beta=0.25$, CI95\% [0.21; 0.28], $p<0.001)$.

\section{Discussion}

In this international, multicultural sample of young adults, we found that anxiety and depressive symptoms and substance use were associated with IGD symptoms independently of Internet use time, presence of psychiatric diagnosis, culture, or sociodemographic characteristics. Importantly, we found gender differences in the observed associations. Besides extended Internet 
browsing per day for both, more significant IGD symptoms were associated additionally with higher levels of anxiety and depressive symptoms for males, while with marihuana use and higher levels of depressive symptoms for females.

Our study confirmed a higher presence of IGD symptomatology in males compared to females as previously reported [32]. We also showed a differential pattern of associations of IGD symptomatology with anxiety and depressive symptoms and substance use in males and females. In this way, we confirmed a previous study [38] that in a smaller sample found both high anxiety and depressive symptoms to be associated with greater IGD symptoms, where greater symptomatology of IGD in males compared to females, while in females, only depressive symptoms were associated with IGD. Despite the general contributing factors to IGD symptomatology, we observed that IGD symptoms were differently affected by various substance use in males and females. The general association of IGD and substance use is in line with a recent review [28], which concluded that many of the problematic behaviors co-occurring with disordered gaming can be performed concurrently with problematic gaming, potentially as part of a coping strategy [45]. Unique associations between various substance use, psychological symptoms, and IGD found in our study for males and females might indicate possible gender-specific predisposition, coping strategies, or vulnerabilities, what may be used in therapeutic approaches to tackle IGD symptom severity as gender specific. These gender differences suggest that IGD may be associated with a "stimulation" experience of using substances in females (as revealed by a positive association between stimulant drugs and absence of association between depressant drugs and IGD symptoms) and a "depressive" in males (as revealed by the negative association between stimulant drugs and IGD symptoms and a positive association between depressant drugs and IGD symptoms). This is consistent with evidence showing that actions occurring for natural rewards and that there are common and specific underpinnings for the associations between drug use and gaming disorder. Future studies that clarify neurobiology [46] and coping strategies [45] explain gender differences in IGD. The available literature suggests the possible gender-specific association between cannabis use and depressive symptoms, with females showing stronger associations between cannabis use and depression than males [47]. However, surveys consistently show that anxiety disorders are more common among females [48] and females may be more prone than males to self-medicate for mood problems with substances such as alcohol [49]; moreover, females are more likely to drink to regulate negative affect and stress reactivity $[50,51]$. Our findings suggest that specific drug use may strengthen the impact of symptoms of anxiety and depression on IGD. These patterns of associations need to be studied further; however, they are in line with the current knowledge on differences in gender-specific mechanisms of alcohol and cannabis effects. Encountering substance use severity and its relationship to the prevalence of anxiety/depression symptoms is an important future direction for developing gender-appropriate interventions to address IGD.

The results of our study should be interpreted with caution due to some relevant limitations. First, we evaluated the relationship between symptoms of anxiety and depression only on IGD. An association of IGD/ problematic Internet use and impulsivity is frequently reported [52-54]. For example, Ryu et al. [55] showed that high impulsivity affected depressive symptoms and increased the risk of IGD. Thus, future studies should evaluate impulsivity alongside symptoms of anxiety and depression. Whether the currently observed effect is the same in other types of stress-related conditions, personality traits [56], emotional intelligence [15], or other traits/motives such as escapism [57] is unknown. Another major limitation is that we used a cross-sectional study design, and to demonstrate causal effects, prospective studies are needed and clinical trials on the interventions addressing both substance use and anxiety and depression management. In addition, only students who agreed to participate were included, and that the response rates varied substantially between countries. Furthermore, the sampling was convenient, and those who may have different or more pronounced patterns of IGD might not have participated. In addition, we equated a country-level with culture-level, which may not be sufficient to represent culture, and we could not obtain enough data for other sub-cultural and sub-racial groups, and we were not collecting data on Internet access availability, equipment used, and legal conditions of some substances' availability, which could limit the generalizability of the findings. Finally, only self-reports were used to assess psychiatric symptoms, and respective diagnoses were not confirmed since it was not able to provide clinical assessments to the participants.

\section{Conclusions}

Our study found that more overt symptoms of IGD were associated with higher levels of anxiety and depressive symptoms and substance use. Still, these associations may differ among males and females, what should be considered in therapeutic approaches targeting IGD. Factors mediating the 
relationship between mental distress and IGD symptoms might not be the same as contributing factors. Therefore, a better understanding of mechanisms related to psychiatric symptoms contributing to IGD symptoms is warranted.

\section{Abbreviations}

ICD: International Classification of Diseases; ICMH-SG: International Child Mental Health - Study Group; ICMH-IGD: ICMH-Internet Gaming Disorder Selfreport for College/University Students; IGD: Internet gaming disorder; DSM: Diagnostic and Statistical Manual; GAD-7: Generalized Anxiety Disorder scale7; PHQ-9: Patient Health Questionnaire-9; SEM: Structural equation modeling; UAE: United Arab Emirates.

\section{Supplementary Information}

The online version contains supplementary material available at https://doi. org/10.1186/s43045-022-00180-6.

\section{Additional file 1.}

\section{Acknowledgements}

This study was organized by the International Child Mental Health - Study Group (ICMH-SG), which is committed to advancing cross-cultural research across various aspects of mental health (https://www.icmhsg.org/). The authors would like to thank to Jelena Jović, Ivana Tadic, Olayinka Atilola, Mohamad Avicenna, Pedro de Abreu Monteiro Campos, Cristine Moreira, Jose Carlos Tavares, and Massimiliano Buoli who help collecting data.

\section{Authors' contributions}

$A D, Y P S B$, and $D S$ were responsible for organizing the project. YPSB, JB, IGB, AS, RR, TWT, TNH, HY, GE, ZV, MRRC, PS, RS, INM, LAMC, ARB, RK, and DS contributed to organizing the study, its data collection, and interpretation for the individual countries. JB, PM, and SF analyzed and interpreted all data together. JB and IGB wrote the first draft of the manuscript. All authors contributed significantly in the revisions of the manuscript and all read and approved the final manuscript submitted.

\section{Funding}

$J B$ and IGB has received funding from the Research Council of Lithuania (LMTLT), project agreement No S-GEV-20-5, while no other funds were received.

\section{Availability of data and materials}

The datasets used and/or analyzed during the current study are available from the corresponding author on reasonable request.

\section{Declarations}

\section{Ethics approval and consent to participate}

The informed consent was obtained from all the participants and the study protocol was approved by the institute ethical board/committee of respective authors'institution prior to the start of data collection.

\section{Consent for publication}

Not applicable.

\section{Competing interests}

None of the authors declare any conflict of interests except Julius Burkauskas, who has been working as consultant at Cogstate, Ltd., over the past several years.

\section{Author details}

'Laboratory of Behavioral Medicine, Neuroscience Institute, Lithuanian University of Health Sciences, Vyduno al 4, Palanga, Lithuania. ${ }^{2}$ Department of Neurobiology and Biophysics, Vilnius University, Vilnius, Lithuania. ${ }^{3}$ University of Rijeka, Rijeka, Croatia. ${ }^{4}$ Behavioral Addictions Clinic (BAC), Department of Psychiatry and National Drug Dependence Treatment Center (NDDTC),
All India Institute of Medical Sciences (AllMS), New Delhi, India. ${ }^{5}$ Centre for Addiction Medicine, National Institute of Mental Health and Neuroscience, Bengaluru, India. ${ }^{6}$ Department of Psychiatry, B K L Walawalkar Rural Medical College, Sawarde, Maharashtra, India. ${ }^{7}$ South Vietnam HIV Addiction Technical Transfer Centre - University of Medicine and Pharmacy, Ho Chi Minh City, Vietnam. ${ }^{8}$ Faculty of Public Health, University of Medicine and Pharmacy, Ho Chi Minh City, Vietnam. ${ }^{9}$ Ankara Children's Hematology and Oncology Training and Research Hospital Department of Child and Adolescent Psychiatry, Ankara, Turkey. ${ }^{10}$ Diskapi Training and Research Hospital, Ankara, Turkey. ${ }^{11}$ Department of Psychology \& Counseling, United Arab Emirates University, Al Ain, United Arab Emirates. ${ }^{12}$ United Nations Department of Safety and Security, Cox's bazar, Bangladesh. ${ }^{13}$ Department of Psychiatry, Patan Academy of Health Sciences, School of Medicine, Lalitpur, Nepal. ${ }^{14}$ Universidade Lusíada Norte (Porto); Centro de investigação em Psicologia para o Desenvolvimento (CIPD), Porto, Portugal. ${ }^{15}$ Psychiatry Department, Dr Soeharto Heerdjan Mental Hospital Jakarta, Jakarta, Indonesia. ${ }^{16}$ Universidade Católica de Petrópolis Rio de Janeiro, Rio de Janeiro, Brazil. ${ }^{17}$ Department of Psychiatric Rehabilitation, Department of Psychiatry and Psychotherapy, Medical University of Silesia, Katowice, Poland. ${ }^{18}$ Clinic for Neurology and Psychiatry for Children and Youth, Dr. Subotic 6a, Belgrade 11000, Serbia.

Received: 13 December 2021 Accepted: 16 January 2022

Published online: 14 February 2022

\section{References}

1. Derevensky JL, Richard J (2020) Response to commentary: the future of gaming disorder research and player protection: what role should the video gaming industry and researchers play. Int J Ment Health Addict 18:800-805

2. Griffiths MD, Pontes HM (2020) The Future of gaming disorder research and player protection: what role should the video gaming industry and researchers play? Int J Ment Health Addict 18:784-790

3. Shi J, Potenza MN, Turner NE (2020) Commentary on: "The future of gaming disorder research and player protection: what role should the video gaming industry and researchers play?". Int J Ment Health Addict 18:791-799

4. American Psychiatric Association (2013) Diagnostic and statistical manual of mental disorders (DSM-5). American Psychiatric Publishing, Arlington

5. World Health Organization (WHO) (2018) International statistical classification of diseases and related health problems (11th Revision; ICD-11). Retrieved from: https://icd.who.int/browse11//-m/en.

6. King DL, Delfabbro PH, Potenza MN, Demetrovics Z, Billieux J, Brand M (2018) Internet gaming disorder should qualify as a mental disorder. Aust N Z J Psychiatry 52:615-617

7. Chamberlain SR, Grant JE (2019) Behavioral addictions. A transdiagnostic approach to obsessions, compulsions and related phenomena, p 401

8. Kardefelt-Winther D, Heeren A, Schimmenti A, van Rooij A, Maurage P, Carras M et al (2017) How can we conceptualize behavioural addiction without pathologizing common behaviours? Addiction 112:1709-1715

9. Pornnoppadol C, Ratta-apha W, Chanpen S, Wattananond S, Dumrongrungruang N, Thongchoi K et al (2020) A comparative study of psychosocial interventions for internet gaming disorder among adolescents aged 13-17 years. Int J Ment Health Addict 18:932-948

10. Torres-Rodríguez A, Griffiths MD, Carbonell X, Farriols-Hernando N, Torres-Jimenez E (2019) Internet gaming disorder treatment: a case study evaluation of four different types of adolescent problematic gamers. Int J Ment Health Addict 17:1-12

11. Zajac K, Ginley MK, Chang R (2020) Treatments of internet gaming disorder: a systematic review of the evidence. Exp Rev Neurother 20:85-93

12. González-Bueso V, Santamaría JJ, Fernández D, Merino L, Montero E, Ribas $J$ (2018) Association between internet gaming disorder or pathological video-game use and comorbid psychopathology: a comprehensive review. Int J Environ Res Public Health 15:668

13. Wichstrøm L, Stenseng F, Belsky J, von Soest T, Hygen BW (2019) Symptoms of internet gaming disorder in youth: predictors and comorbidity. J Abnorm Child Psychol 47:71-83

14. Delfabbro PH, McArdle P, King DL (2020) Political and policy issues in adolescent addiction. In: Essau C, Delfabbro P (eds) Adolescent addiction, 2nd edn. Elsevier, New York 
15. Kircaburun K, Demetrovics Z, Griffiths MD, Király O, Kun B, Tosuntaş ŞB (2020) Trait emotional intelligence and internet gaming disorder among gamers: the mediating role of online gaming motives and moderating role of age groups. Int J Ment Health Addict 18:1446-1457

16. Bányai F, Zsila Á, Kökönyei G, Griffiths MD, Demetrovics Z, Király (2021) The moderating role of coping mechanisms and being an e-sport player between psychiatric symptoms and gaming disorder: Online Survey. JMIR Ment Health 8:e21115

17. Fineberg NA, Demetrovics Z, Stein DJ, loannidis K, Potenza MN, Grunblatt E et al (2018) Manifesto for a European research network into problematic usage of the Internet. Eur Neuropsychopharmacol 28:1232-1246

18. Saletti SM, Van den Broucke S, Chau C (2021) The effectiveness of prevention programs for problematic Internet use in adolescents and youths: a systematic review and meta-analysis. Cyberpsychology 15(2):10

19. Király O, Potenza MN, Stein DJ, King DL, Hodgins DC, Saunders J et al (2020) Preventing problematic internet use during the COVID-19 pandemic: consensus guidance. Compr Psychiatry 100:152180

20. Fineberg NA, Baldwin DS, Menchon JM, Denys D, Grunblatt E, Pallanti S et al (2013) Manifesto for a European research network into obsessive-compulsive and related disorders. Eur Neuropsychopharmacol 23:561-568

21. Choi J, Cho H, Kim JY, Jung DJ, Ahn KJ, Kang HB et al (2017) Structural alterations in the prefrontal cortex mediate the relationship between Internet gaming disorder and depressed mood. Sci Rep 7:1-10

22. Li L, Yao YW, Li CSR, Zhang JT, Xia CC, Lan J et al (2018) The comorbidity between internet gaming disorder and depression: Interrelationship and neural mechanisms. Front Psychiatry 9:154

23. Teng Z, Pontes MH, Nie Q, Griffiths MD, Guo C (2021) Depression and anxiety symptoms associated with internet gaming disorder before and during the COVID-19 pandemic: a longitudinal study. J Behav Addict 10:169-180

24. Sepede G, Tavino M, Santacroce R, Fiori F, Salerno RM, Di Giannantonio M (2016) Functional magnetic resonance imaging of internet addiction in young adults. World J Radiol 8:210

25. Wan HR, Cho H, Dj K (2018) Prevalence and correlates of comorbid depression in a nonclinical online sample with DSM-5 internet gaming disorder. J Affect Disord 226:1-5

26. Wang CY, Wu YC, Su CH, Lin PC, Ko CH, Yen JY (2017) Association between Internet gaming disorder and generalized anxiety disorder. J Behav Addict 6:564-571

27. Wartberg L, Kriston L, Thomasius R (2017) The prevalence and psychosocial correlates of Internet gaming disorder. Dtsch Arztebl Int 114:419-424

28. Burleigh TL, Griffiths MD, Sumich A, Stavropoulos V, Kuss D (2019) A systematic review of the co-occurrence of gaming disorder and other potentially addictive behaviors. Cur Addict Rep 6:383-401

29. Brand M, Young KS, Laier C (2014) Prefrontal control and Internet addiction: a theoretical model and review of neuropsychological and neuroimaging findings. Front Hum Neurosci 8:375

30. Dong GH, Wang M, Wang Z, Zheng H, Du X, Potenza MN (2020) Addiction severity modulates the precuneus involvement in internet gaming disorder: functionality, morphology and effective connectivity. Prog Neuropsychopharmacol Biol Psychiatry 98:109829

31. Kuss DJ (2013) Internet gaming addiction: current perspectives. Psychol Res Behav Manag 6:125

32. Kuss DJ, Griffiths MD (2012) Internet and gaming addiction: a systematic literature review of neuroimaging studies. Brain Sci 2:347-374

33. Su W, Han X, Yu H, Wu Y, Potenza MN (2020) Do men become addicted to internet gaming and women to social media? A meta-analysis examining gender-related differences in specific internet addiction. Comput Hum Behav 113:106480

34. Dong GH, Wang M, Wang Z, Du X, Potenza MN (2019) Gender-related functional connectivity and craving during gaming and immediate abstinence during a mandatory break: implications for development and progression of internet gaming disorder. Prog Neuropsychopharmacol Biol Psychiatry 88:1-10

35. Hof RA, Howell JC, Wampler J, Krishnan-Sarin S, Potenza MN (2020) Differences in associations between problematic video-gaming, videogaming duration, and weapon-related and physically violent behaviors in adolescents. J Psychiatr Res 121:47-55

36. Barua A, Barua A (2012) Gendering the digital body: women and computers. Al Soc 27:465-477
37. Li Q, Dai W, Zhong Y, Wan L, Dai B, Liu X (2019) The mediating role of coping styles on impulsivity, behavioral inhibition/approach system, and internet addiction in adolescents from a gender perspective. Front Psychol 10:2402

38. Bonnaire C, Baptista D (2019) Internet gaming disorder in male and female young adults: the role of alexithymia, depression, anxiety and gaming type. Psychiatry Res 272:521-530

39. Balhara YPS, Doric A, Stevanovic D, Knez R, Singh S, Chowdhury M et al (2019) Correlates of problematic Internet use among college and university students in eight countries: an international cross-sectional study. Asia J Psychiatr 45:113-120

40. Stevanović D, Đorić A, Balhara YPS, Ćirović N, Arya S, Ransing R et al (2020) Assessing the symptoms of Internet gaming disorder among college/university students: an international validation study of a selfreport. Psihologija 53:43-63

41. Pontes HM, Stavropoulos V, Griffiths MD (2017) Measurement invariance of the internet gaming disorder scale-short-form (IGDS9-SF) between the United States of America, India and the United Kingdom. Psychiatr Res 257:472-478

42. King DL, Chamberlain SR, Carragher N, Billieux J, Stein D, Mueller K et al (2020) Screening and assessment tools for gaming disorder: a comprehensive systematic review. Clin Psychol Rev 77:101831

43. Spitzer RL, Kroenke K, Williams JB, Lowe B (2006) A brief measure for assessing generalized anxiety disorder: the GAD-7. Arch Intern Med 166:1092-1097

44. Spitzer RL, Kroenke K, Williams JB (1999) Validation and utility of a self-report version of PRIME-MD: the PHQ primary care study. Primary Care Evaluation of Mental Disorders. Patient Health Questionnaire. JAMA 282:1737-1744

45. Na E, Lee H, Choi I, Kim DJ (2017) Comorbidity of Internet gaming disorder and alcohol use disorder: a focus on clinical characteristics and gaming patterns. Am J Addict 26:326-334

46. Althaus J, Zendle D, Bowden-Jones H (2021) Gambling and gaming addictions in women. el-Guebaly N, Carrà G, Galanter M, Baldacchino AM (Eds) Textbook of addiction treatment. Springer, Cham.

47. Re JM, Sawyer MG, Raphae B, Patton GC, Lynskey M (2002) Mental health of teenagers who use cannabis: results of an Australian survey. Br J Psychiatry 180:216-221

48. Smit JP, Randall CL (2012) Anxiety and alcohol use disorders: comorbidity and treatment considerations. Alcohol Res 34:414-431

49. Brady KT, Randall CL (1999) Gender differences in substance use disorders. Psychiatr Clin North Am 22:241-252

50. Beccaria, Berger S, Lopez T (2019) Alcohol as a coping mechanism for social anxiety. J Addict Psychol 3

51. Peltier M, Verplaetse TL, Mineur YS, Petrakis I, Cosgrove KP, Picciotto MR, McKee SA (2019) Sex differences in stress-related alcohol use. Neurobiol Stress 10:100149

52. Blinka L, Skarupová K, Mitterová K (2016) Dysfunctional impulsivity in online gaming addiction and engagement. Cyberpsychology 10:5

53. Gecaite-Stonciene J, Saudargiene A, Pranckeviciene A, Liaugaudaite V, Griskova-Bulanova I, Simkute D et al (2021) Impulsivity mediates associations between problematic internet use, anxiety, and depressive symptoms in students: a cross-sectional COVID-19 study. Front Psychiatry 12:17

54. Şalvarlı ŞI, Griffiths MD (2019) The association between internet gaming disorder and impulsivity: a systematic review of literature. Int J Ment Health Addict:1-27. https://doi.org/10.1007/s11469-019-00126-w

55. Ryu H, Lee JY, Choi A, Park S, Kim DJ, Choi JS (2018) The relationship between impulsivity and internet gaming disorder in young adults: mediating effects of interpersonal relationships and depression. Int J Environ Res Public Health 15:458

56. Şalvarlı Şı, Griffiths MD (2019) Internet gaming disorder and its associated personality traits: a systematic review using PRISMA guidelines. Int J Ment Health Addict 19:1420-1442

57. Šporčić B, Glavak-Tkalj R (2018) The relationship between online gaming motivation, self-concept clarity and tendency toward problematic gaming. Cyberpsychology 12:4

\section{Publisher's Note}

Springer Nature remains neutral with regard to jurisdictional claims in published maps and institutional affiliations. 